\title{
A fungus in the haystack
}

\section{An unusual pediatric case of otomycosis from a rural farming area}

Eric Roszell (Meds 2016), Craig Olmstead (Meds 2017)

Faculty Reviewer: Dr Michael John, MD, FRCPC (Department of Medicine, Division of Infectious Diseases)

\section{INTRODUCTION}

When medical professionals are presented with a new patient, the likely diagnoses are worked through first before some of the rarer diagnoses, the zebras, are fully considered. What is important to consider in the domain of rural medicine is that what may be uncommon in big cities is common in a rural setting. In this edition of the zebra files, we are introduced to a case that exemplifies this idea.

\section{CASE PRESENTATION}

Our zebra case begins with a 17-year-old Caucasian female that presented to pediatric acute care with a 3-day history of right ear pain. ${ }^{1}$ When interviewed, she denied any recent airplane rides, loud noises, swimming, or using cotton buds to clean her ears. Other pertinent negatives included no history of nausea, dizziness, or upper respiratory symptoms. She did, however, complain of decreased hearing in her right ear, as well as ear drainage and pain upon pinna manipulation. Otoscopy was performed and revealed a normal tympanic membrane. Direct observations of inflammation were not explicitly recorded at the time of initial presentation. Based on these findings a diagnosis of otitis externa, or inflammation of the external auditory canal, was made and the patient was started on oral ciprofloxacin as well as ciprofloxacin-hydrocortisone otic drops. It should be noted that topical agents are usually sufficient for the treatment of uncomplicated otitis externa; oral ciprofloxacin is usually reserved for those at risk of malignant otitis externa. For most patients in this age group presenting with this constellation of symptoms, antibiotic treatment of the bacteria underlying an otitis externa would end the case here. ${ }^{2}$

Our zebra case, however, was not resolved so quickly. The patient returned to the clinic 9 days later complaining of worsening right-sided hearing loss. Physical examination revealed a right ear canal occluded with cerumen (earwax). Curettage and irrigation of the external auditory canal were performed to remove the excess wax, and the patient reported improved hearing. Five days later the patient presented again to the emergency room, complaining of right ear discomfort and hearing difficulties. Upon repeat curettage and irrigation, purulent material and debris were removed. A neomycin/polymyxin/hydrocortisone otic suspension was started and the patient was asked to follow up in pediatrics. Five days later, the patient still reported trouble hearing with the right ear. At this time, it was noted that our zebra patient had several moist pieces of hay in her hair and further history revealed that the patient had been baling hay on a farm all summer. On physical examination, a white mucoid material was visualized on the right tympanic membrane. A culture of this material was obtained, resulting in an identification of the fungus, Aspergillus niger, and a diagnosis of otomycosis was confirmed.

\section{OTOMYCOSIS}

Otomycosis, or a fungal infection of the ear, can be caused by many different fungal varieties, most commonly of the Aspergillus or Candida genera., ${ }^{3,4}$ Almost all patients with otomycosis present with pruritus of the ear; ${ }^{4}$ otalgia, otorrhea, and hearing loss are also common symptoms. ${ }^{3,4}$ In healthy patients, such fungal infections are generally superficial in nature, presenting with chronic otitis externa. ${ }^{4}$ In immunocompromised patients otomycosis can progress beyond the outer ear, causing perforation of the eardrum and in rare cases spread of infection to the middle ear or even to the meninges or mastoid process. Being fungal in origin, otomycosis is expectedly unresponsive to antibiotics. ${ }^{3}$ Swimming, ${ }^{1,3}$ exposure to polluted water, ${ }^{3}$ the presence of skin-compromising conditions such as psoriasis, ${ }^{3}$ as well as recent use of aural antibiotics are associated with otomycosis.,

Diagnosis is based on history, physical exam, and visualization of the external ear. ${ }^{3}$ If necessary, laboratory tests including cultures can be employed to confirm that the nature of the infection is indeed fungal and to determine its etiology. ${ }^{3,5}$ Where there is suspicion of more advanced infection, both computed tomography (CT) of the head and nuclear medicine bone scans can help characterize the extent of disease by demonstrating destructive changes to nearby cranial bones, ${ }^{3,5}$ while enzyme-linked immunosorbent assays (ELISA) can detect fungal antigen in the serum and cerebrospinal fluid. ${ }^{3}$

When the infection is confined to the outer ear, treatment of otomycosis typically involves mechanical removal of fungal material from the ear in addition to administration of topical antifungal agents. $^{1,3}$ The specific topical antifungal used should depend on the organism responsible for the infection. ${ }^{3}$ Agents that work against the most common fungi responsible for otomycosis, namely those of the Aspergillus or Candida genera, are therefore preferred. ${ }^{6}$ Ketoconazole, clotrimazole, and micozanole are examples of appropriate topical antifungals. ${ }^{3,6}$

When the infection has progressed beyond the outer ear, systemic antifungal treatments are necessary. Orally-administered antifungals such as itraconazole and voriconazole function well against both Aspergillus and Candida infections, ${ }^{3}$ with voriconazole demonstrating particular effectiveness against Aspergillus niger ${ }^{6}$ and invasive fungal infections. ${ }^{3}$

\section{RETURNING TO THE CASE}

Culture of the exudates from our patient's ear canal revealed $A s$ pergillus niger. Aspergillus niger is a common fungus found throughout the world, ${ }^{4}$ known to cause black mold growth on certain fruits and vegetables, particularly onions. ${ }^{7}$ This fungus grows most readily in hot and humid climates, ${ }^{7}$ and the ear canal provides a suitable environment for its growth, particularly after exposure to water., ${ }^{3,4}$ This 


\section{ZEBRA FILES}

patient's time spent baling moist hay was suspected as a contributing factor to her otomycosis. ${ }^{1}$ The patient was subsequently treated with oral itraconazole, which resolved the infection. The usage of an oral agent instead of a topical agent may suggest that there was a concern that malignant otitis externa would develop in our patient; however, the specific reasons leading to this clinical decision were not recorded. Generally, oral agents are only used in uncomplicated otomycosis patients after topical agents have failed to resolve the infection. ${ }^{6}$

This case is unusual due to the conditions that led to the episode of otomycosis. Although otomycosis is not rare, as 9 to $25 \%$ of otitis externa is caused by fungi, it is generally not found without multiple risk factors and is more common in tropical countries. ${ }^{1}$ Most cases are associated with immunodeficiency, swimming, topical steroids, or other fungal skin infections, which were all determined to not be factors in our zebra patient. ${ }^{1}$ Our patient did, however, have some risk factors: recent antibiotic use and exposure to farming activities. It has been reported in the literature that farmers are exposed to high levels of molds and fungi during hay- or grain-handling activities. ${ }^{1}$ Although it has not been previously reported, this case of otomycosis is believed to have originated from fungal exposure during hay baling by our zebra patient. Furthermore, the use of antibiotics may have exacerbated the initial fungal colonization. This case suggests that otomycosis should be considered in rural farming communities when patients present with otalgia and otorrhea. As a result, this case demonstrates how in rural medicine practices, one must consider how unique exposures can lead to diagnoses that are not seen in larger centres.

\section{REFERENCES}

1. Aswani V, ShuKla SK. Two unusual pediatric cases of fungal infections in farming families. J Agromedicine. 2011 Apr;16(2):153-7.

2. Piercefield EW, Collier SA, Hlavsa MC, Beach MJ. Estimated burden of acute otitis externa--United States, 2003-2007. Morb Mortal Wkly Rep. 2011 May;60(19):605-9.

3. Vennewald I, Klemm E. Otomycosis: diagnosis and treatment. Clin Dermatol. 2010 Mar;28(2):202-11.

4. García-Agudo L, Aznar-Marín P, Galán-Sánchez F, García-Martos P, Marín-Casanova P, Rodríguez-Iglesias M. Otomycosis due to filamentous fungi. Mycopathologia. 2011 Oct;172(4):307-10.

5. Hsu C, Chen C, Wang H. Iatrogenic invasive otomycosis. Tzu Chi Med J. 2011;23(2):66-8.

6. Ho T, Vrabec JT, Yoo D, Coker NJ. Otomycosis: clinical features and treatment implications. Otolaryng Head Neck. 2006 Nov;135(5):787-91.

7. El-Nagerabi SAF, Ahmed AHM. Storability of onion bulbs contaminated by Aspergillus niger mold. Phytoparasitica. 2003 Oct;31(5):515-23. 